\title{
Design of a dietary intervention to assess the impact of a gluten-free diet in a population with type 1 Diabetes and Celiac Disease
}

\author{
Esther Assor ${ }^{1 *}$, Margaret A. Marcon², Natasha Hamilton ${ }^{3}$, Marilyn Fry ${ }^{4}$,Tammy Cooper ${ }^{5}$ and Farid H. Mahmud ${ }^{1}$
}

\begin{abstract}
Background: Celiac Disease occurs at a 5-10 fold greater prevalence in patients with type-1 diabetes (T1D), despite this increased risk, there is limited objective evidence regarding the impact of a Gluten-Free Diet (GFD) in the large proportion of asymptomatic (30-70 \%) patients with both autoimmune diseases. Given the requirements and intricacies inherent to each condition, we describe the rationale and design a dietary curriculum specifically addressing the educational requirements for children and adults with CD and diabetes as part of the CD-DIET Study.
\end{abstract}

Methods and design: The CD-DIET Study (Celiac Disease and Diabetes - Dietary Intervention and Evaluation Trial) is a multicenter randomized controlled trial aimed at evaluating the safety and efficacy of a GFD in patients with asymptomatic celiac disease and T1D on key diabetes and patient-centered outcomes.

Discussion: Key dietary components of the trial include a description and evaluation of food consumption patterns including glycemic index and glycemic load, novel assessments of gluten quantification, and objective and subjective measures of GFD adherence. This dietary curriculum will establish rigorous guidelines to assess adherence and facilitate evaluation of a GFD on metabolic control, bone health and patient quality of life in patients with CD and diabetes.

Trial registration Number: NCT01566110. Date of Registration: March, 2012.

\section{Background}

Celiac Disease (CD) is an autoimmune disease characterized by damage to the small intestine, triggered by gluten ingestion from wheat, rye and barley and their derivatives in genetically susceptible individuals $[1,2]$. Currently the only treatment for $\mathrm{CD}$ is a lifelong adherence to the gluten-free diet. CD prevalence in T1D patients approximates 5-7 \%. Despite this increased risk, many health care professionals struggle as to the optimal approach to managing CD in Type 1 Diabetes (T1D). The diagnosis of patients who present with symptomatic $\mathrm{CD}$, including malabsorption and obvious pathology upon biopsy, remains straightforward, with improvements noted on a

\footnotetext{
* Correspondence: esther.assor@sickkids.ca

'Department of Pediatrics, Division of Endocrinology, Hospital for Sick

Children, University of Toronto, Toronto, ON, Canada

Full list of author information is available at the end of the article
}

gluten-free diet. Many patients identified by screening, however, tend to be asymptomatic [3, 4]. Evidence is inconclusive as to whether the benefits of screening and potentially treating asymptomatic individuals outweigh the harms of managing a population already burdened with an established chronic illness.

The CD-DIET Study (Celiac Disease and Diabetes Dietary Intervention and Evaluation Trial) is a multicenter randomized controlled trial aimed at evaluating the safety and efficacy of a GFD in patients with asymptomatic celiac disease and T1D over 1 year. Key outcomes include the impact of GFD on diabetes control (HbA1c), bone health, blood glucose variability, and patient centered measures including quality of life and self-perceived health. Dietary outcomes will include description and evaluation of food consumption patterns including glycemic index and load, dietary characteristics including gluten quantification, and 
highlight objective and subjective measures of GFD adherence. As a consequence, a de-novo dietary curriculum specifically addressing the educational requirements for GFD and T1D was developed to study dietary adherence and facilitate evaluation of key dietary components and their impact on study outcomes (Fig. 1).

An additional challenge of this trial relates to potential difficulties with individual adherence to their assigned dietary group. As such, significant effort was invested in a patient and family centered dietary experience supported by dietitians with extensive knowledge of diabetes, and a curriculum with subjective and objective assessment for dietary quality and gluten content. A de novo study dietary handbook was also created for the study, with sections focusing on adaption to the GFD highlighting situational approaches and providing gluten-free diabetes-specific nutritional tables for carbohydrates and glycemic index. This paper describes approach and details of the dietary curriculum implemented as part of the CD-DIET study to address the needs and challenges of living with asymptomatic CD and T1D.

The challenge of T1D \& CD: dietary quality and education $\mathrm{CD}$ is a unique condition since dietary modification is the only currently recommended and effective treatment. Type 1 diabetes is a challenging condition requiring daily efforts to balance meals, activity, and insulin to maintain adequate metabolic control. It is important to assess the impact of $\mathrm{CD}$ on dietary quality and composition as transition to a GFD may pose a risk of nutritional imbalances and/or deficiencies. Dietary inadequacies have been reported to be common with $C D$. This may be inherent

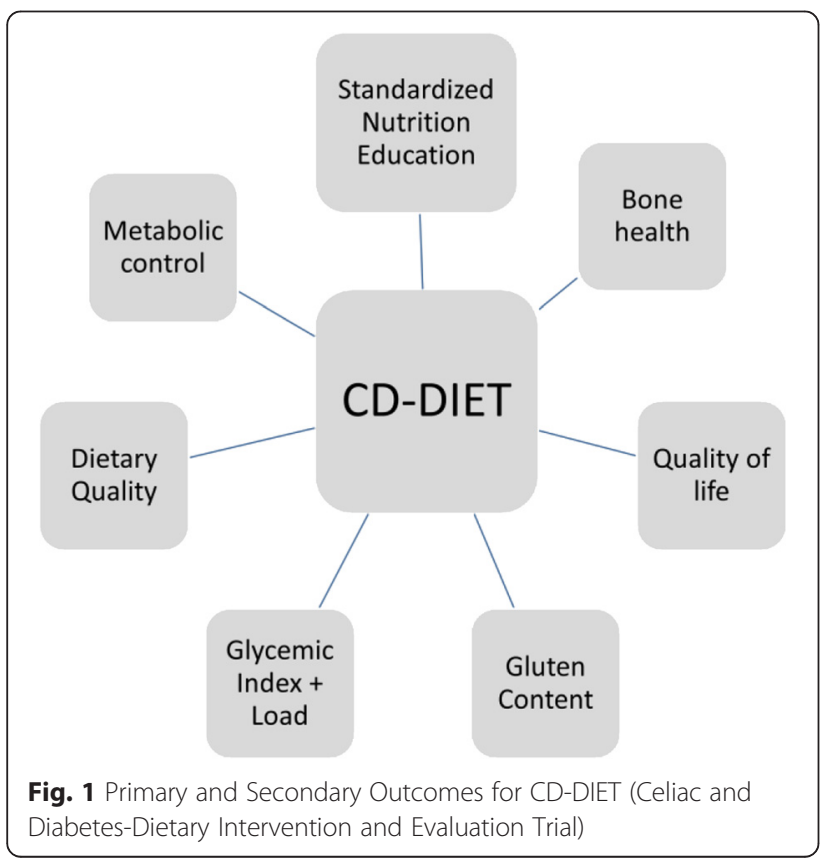

to the diet but also as a result of habitual poorer choices [5-7], although it has been shown that imbalances from the GFD are no different than the imbalances noted in the healthy population [8]. Gluten-free foods available in Canada, analyzed for macro/micronutrient composition were found to be lower in fiber, iron, calcium, protein, folate and vitamin D and higher in total carbs, fat and sugar $[5,6,9,10]$ than products containing gluten. Similar imbalances including higher fat, lower fiber and nutrient intakes have been noted in children and adults with T1D $[11,12]$. Compared to their gluten containing equivalents, processed GF foods including breads, cereal bars and pasta have been reported to be higher in total carbohydrates, lower in protein and higher in sodium, saturated fat and cholesterol [13]. Additionally, many starchy GF foods were noted to have variable mineral content and high glycemic index [14]. From a Canadian context, both the Canadian Celiac Association (CCA) and the Canadian Diabetes Association (CDA) offer access to pertinent information including nutritional and financial support strategies for each medical condition. However there are limited all-encompassing dietary advice/ resources catered to needs of individuals living with the double diagnosis of $\mathrm{CD}$ and T1D. A comprehensive literature review including PubMed, Medline, University of Toronto Libraries and various Internet websites investigated what resources are available on the subject. Our research found that the current available literature is limited in providing effective meaningful and tangible resources, tailored to address the short and long term needs and challenges of both conditions, particularly of asymptomatic patients with $\mathrm{CD}$ and T1D. At our center we have a unique experience of having the opportunity to follow this population affected by a double diagnosis of Type 1 diabetes and Celiac Disease, combined with an expertise and specialization in this field. A comprehensive curriculum has been developed to address the perceived and noted challenges as practitioners, patients and the literature.

\section{Impact of CD in T1D: Quality of life (QOL)}

In studies of existing chronic conditions such as diabetes, which involve mandatory daily routines to remain safe, and daily challenges which require problem solving, it is important to evaluate the impact of the double diagnosis (CD and T1D) that may further negatively impact quality of life (QOL). Areas of negative impact in maintaining a GFD have been described as follows: cost maintaining a GFD, and extend to socializing GF including dining out, impact on family, career, travel and religious practices [15-18] and the corresponding negative psychological symptoms and negative emotions $[19,20]$. The benefit of the gluten-free diet (GFD) on QOL in asymptomatic screened-detected $C D$ patients remains controversial [20-23]. Asymptomatic patients with $C D$ report 
better self-perceived health and less concern with their disease prior to dietary modification $[24,25]$, and over half of patients following a GFD for more than 5 years have reported QOL as excellent or very good [26].

Given these challenges and our clinical experience with our population, a key element of the dietary curriculum is to address all the challenges through education. Information is provided in a supportive, encouraging and individualized manner with regular visits where patients are asked to bring in labels and practice problem solving. The Book' Your Guide to Well Being: Managing Celiac Disease and Diabetes' is written in a modular form so that patients and their families could refer to various sections that they find pertinent. The book addresses and provides tangible and concrete guidance on living with Type 1 and $\mathrm{CD}$. It addresses in detail all aspects of social life and provides practical advice for incorporating a GFD while eating out, traveling, celebrations, management at school, university, and the workforce. A resource list including support groups, helpful websites, associations and organizations, a detailed list of GF foods with their macro and micronutrient contents including GI is available to support the practical day to day life of CD and T1D. To limit financial barriers, all patients on a GFD are provided with a monthly stipend for their groceries.

\section{Dietary assignment in a clinical trial: opportunities and challenges}

Symptomatic patients who follow a GFD experience an improvement in symptoms and therefore following a GFD is meaningful. However it is less clear if there are any related benefits to asymptomatic CD patients with T1D who follow a GFD. The CD-DIET Study represents an opportunity to evaluate the impact of a GFD on asymptomatic patients with $\mathrm{CD}$. This is a randomized, controlled, multicenter, dietary intervention study in pediatric and adult diabetes centers across Ontario, Canada, that is based on an intention to treat. The study consists of a comprehensive dietary interview with defined subjective and objective parameters. It is designed to address the educational and psychosocial needs and challenges of living and thriving with both conditions: $\mathrm{CD}$ and T1D.

CD-DIET is designed to evaluate the efficacy and safety of the gluten-free diet (GFD) in patients with asymptomatic biopsy confirmed CD and T1D. Patients (ages 845) will be randomly assigned to a GFD or continue with their usual diabetes gluten containing meal plan [27] (Fig. 2 study flow diagram). Patients will be followed-up every 3 months where Visit 1 is randomization, Visits 2,3,4 and 5 as $3,6,9$, and 12 months respectively from randomization. We anticipate that this may be a challenge as adherence to dietary advice is reported to be the lowest of all treatment types [28] with a range of $36-96 \%$ as strict adherence [15].

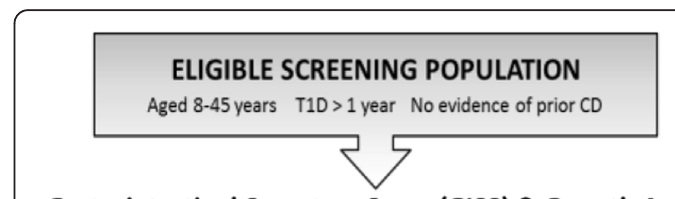

Gastrointestinal Symptom Score (GISS) \& Growth Assessment

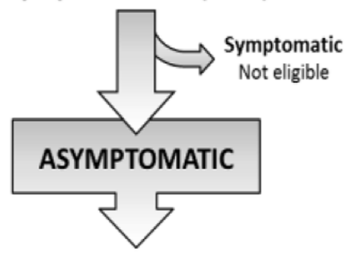

Celiac Serology TTG-IgA Testing \& Anemia Testing

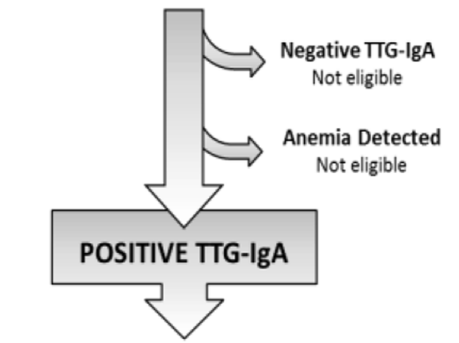

Gastroenterology Consultation

(Symptoms Re-Evaluated)

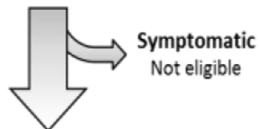

Biopsy Procedure \& Pathologic Review

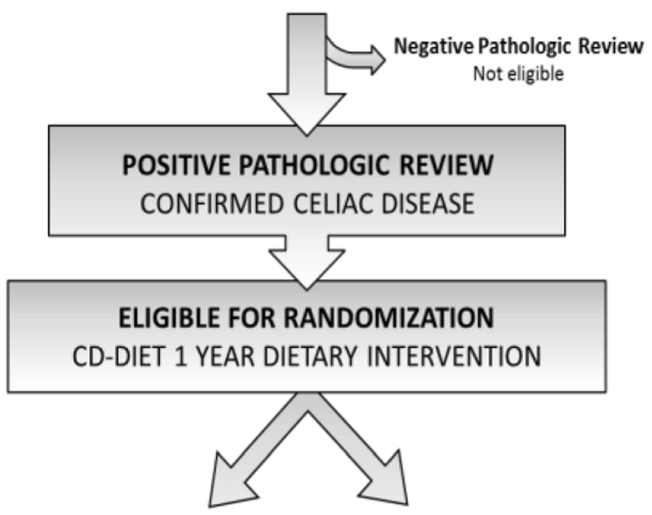

GLUTEN-FREE DIET

GLUTEN-CONTAINING DIET

Fig. 2 Flow diagram illustrating screening and eligibility for Intervention. Mahmud et al BMJ Open 2015

Designing the dietary protocol takes into account 3 key components to successful adherence to the GFD, as identified by the literature and our experience: understanding of the GFD, ability to maintain a GFD while away from home and membership in a celiac support group [23]. These factors not only are met via the guide developed and described above but have been embedded into the dietary curriculum with opportunities to evaluate knowledge and adherence at every visit. Therefore a robust dietary curriculum which includes a detailed 
educational plan of what and when information will be relayed, with opportunities for assessment and evaluation of dietary quality, food consumption patterns and adherence, are key elements to the success of this study.

The dietary curriculum is planned to detect any dietary deficiencies and imbalances of macro and micronutrients as well as dietary fiber, calcium, vitamin D and iron using ESHA, a Food Processor with Nutrition Analysis software. The study will also include gluten consumption patterns of the subjects enrolled using the Osborne formula [29].

"Your guide to Well Being: Managing celiac and Diabetes" was designed specifically for the intervention arm (GFD) to address the intricate educational and social needs of both $\mathrm{CD}$ and diabetes. It was written in a modular form as an easy to use guide with topics ranging from explaining the diagnosis and managing a GF home to special considerations and travel with T1D. Figure 1 depicts an overview of the dietary curriculum with the study visits.

The Role of the dietitian in this study is identified as supporting the subjects to the arm that they are randomized to, but not intended to intensify diabetes management. Subsequently adherence in this study is defined as the ability to follow the prescribed diet. Dietary adherence will be assessed at each clinic visit both objectively and subjectively.

\section{Components dietary assessment and adherence}

3 major components of assessment for adherence were identified which include subjective and objective measures: 1 . a comprehensive dietary interview with alternating 3 day food records and typical day recalls. 2 . Application of Celiac Dietary Adherence test (CDAT) and 3. Serology -TTG IgA titres.

\section{Objective measures of assessment}

The objective measures in the dietary assessments include (Fig. 3):

1. A 3 day food record will be collected and clarified. Subsequently, records will be sent to central analysis where nutrient intake will be analyzed using the computer based ESHA food processor which contains a wide data base capable of analyzing over 160 food nutrient components and compare to DRIs. The analysis will include macronutrient distribution to be compared to CDA practice guidelines and specific nutrients including calcium, iron, vitamin $\mathrm{D}$ and fiber. Alterations in macronutrients, vitamins and minerals have been associated with imbalances and/or deficiencies with the GFD [5-8]. Additionally, Glycemic index (GI) and glycemic load (GL) of mixed meal will be calculated and analyzed for both GFD and GCD.

In this study we are also planning to describe the food consumption patterns of our population with dietary gluten. This will be done by multiplying the plant proteins of overt gluten sources by a factor of 0.8 [29]. This is a relatively novel approach in North America in relation to its application to dietary intake. Our findings from a previous study show that this approach to dietary gluten estimation is reasonable and valid (29). Dietary gluten for the GFD group will be estimated by using the maximum $20 \mathrm{ppm}$ standard as defined by Health Canada's labelling regulations [30].

2. Celiac dietary Adherence Test (CDAT)- a 7 item questionnaire that has been demonstrated to have a highly predictive value ( $88 \%$ ) for verifying compliance with the GFD in adults, as compared to TTG IgA and dietary evaluation [31]. It will be administered to the subjects randomized to the GFD at every visit after randomization (visits 2-5) (Fig. 4).

3. Tissue Transglutaminase IgA Antibody Assay-TTG IgA - we anticipate that if subjects are adherent, serology will normalize or decrease with subjects randomized to the GFD and remain elevated for subjects randomized to the GCD. Blood sample will be taken from both groups at visits 3 and 5 .

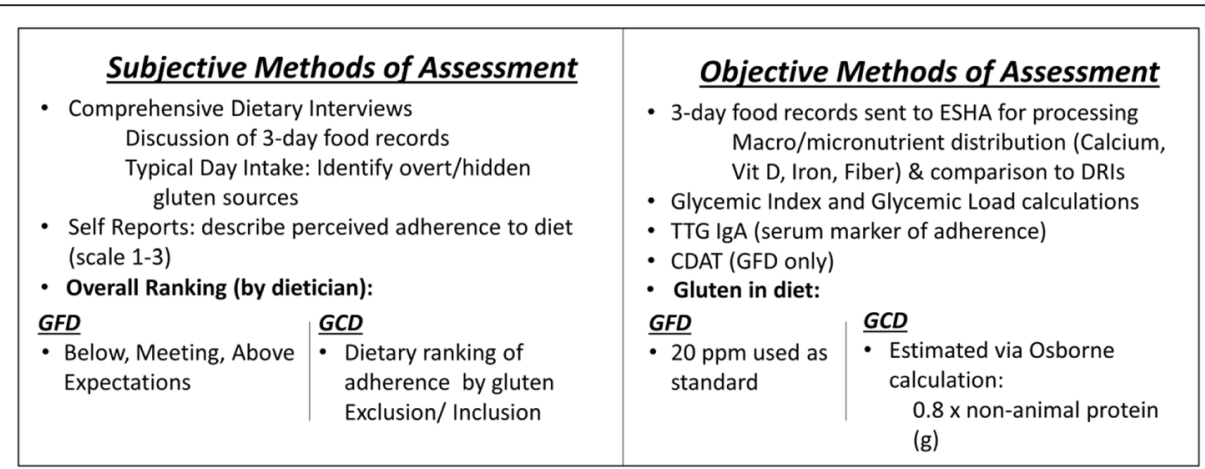

Fig. 3 methods of Assessment for GFD/GCD arms 


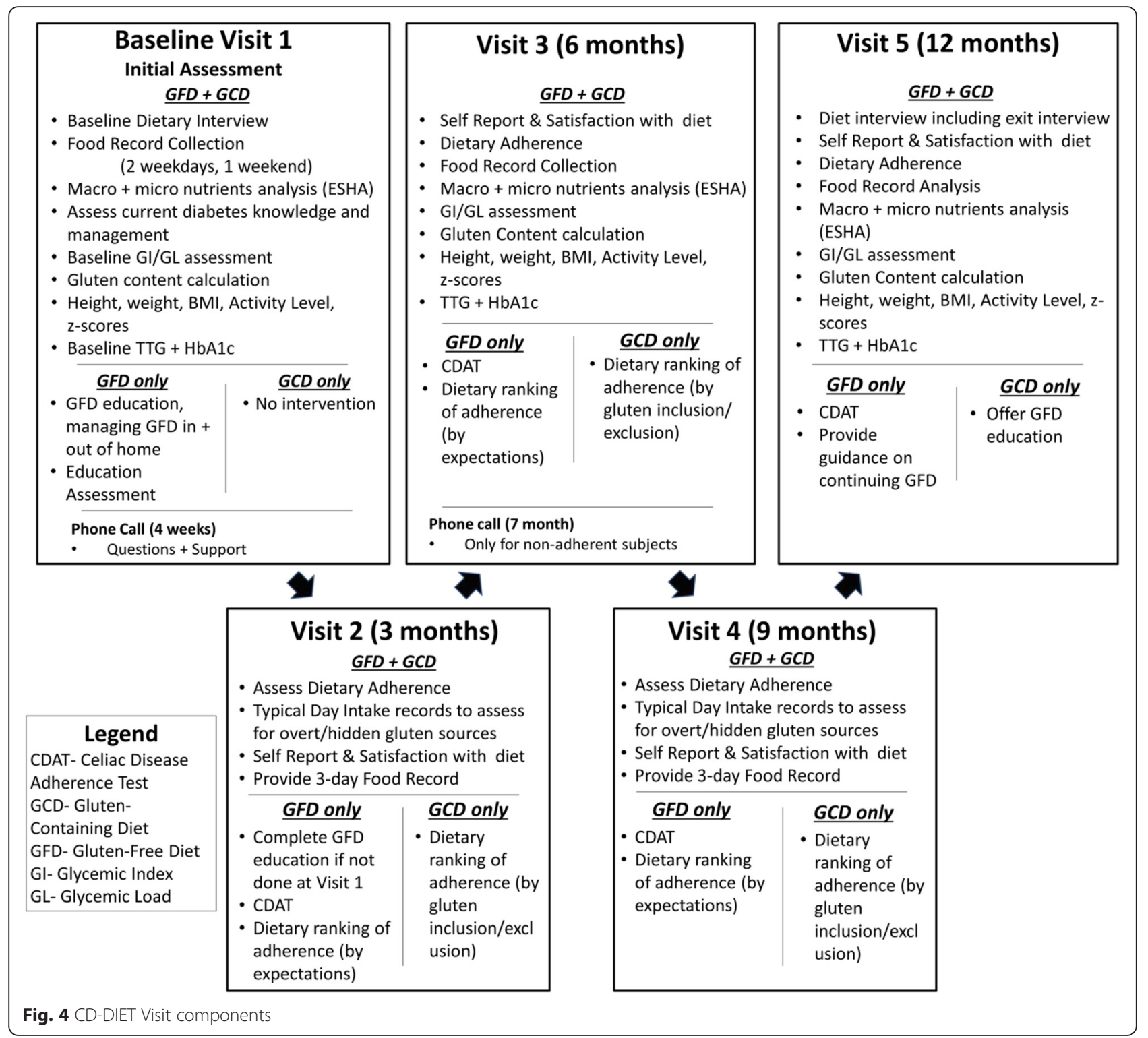

\section{Subjective measures of assessment}

The subjective measures in the dietary assessments include (Fig. 3):

1. Comprehensive dietary interviews to also include 3 day food records (at visits 1, 3 and 5) and a typical day recall (at visits 2,4 ) (Fig. 1 ). In the absence of a 3 day food record a typical day recall will be taken. Through the records, the dietitian will assess for the presence of dietary gluten and/or hidden sources of gluten.

2. Ranking: At the end of every dietary interview a global ranking of adherence will be applied by the dietitian. A ranking of 'expectations' will be applied to the subjects on the GFD arm. The ranking will range from 'Below Expectations' - minimal understanding of GFD concepts and inability to distinguish carbohydrate containing foods, to 'Meeting Expectations' - with the GFD and ability carb count, and 'Above Expectations' - advanced understanding of the GFD, seeks out additional information about GF carb sources and demonstrates an interest in the curriculum. A ranking of gluten inclusion and exclusion will be applied to the GCD arm. The ranking will range from "Complete inclusion of gluten containing (GC) foods to 'Some restriction of GC foods' to a 'strict restriction of GC foods'. 
3. Subject's own self report: Subjects will also have an opportunity to evaluate their own adherence with the diet that they have been randomized to from 'strict' to 'partial' to 'none' adherent.

\section{CD-DIET study dietary overview: methods and design}

Subjects aged 8 to 45 years old with established T1D and biopsy confirmed asymptomatic $\mathrm{CD}$ will be randomized to treatment with GFD for one year or continue with their usual GCD as described (Refer to Figs. 2 and 4) [27].

\section{Dietitian visits}

All subjects on both arms of the study will have five visits with a trained dietitian. Worksheets specific to each arm and visit have been designed to ensure consistent collection of information, and uniform parameters for assessment and education. All worksheets will be filled out by the dietitian at every visit. All participants will fill out food records (two weekdays and 1 weekend day) for visits 1,3 and 5 (at initial assessment, 6 month and 12 months). Food records will be sent to ESHA, and will be reviewed with the participants at their following visit (visits 2 and 4, 3 and 9 months, respectively). Glycemic Index (GI) and Glycemic load (GL) will be calculated to describe the dietary carbohydrates of each arm using glucose as a reference. GI of mixed meals per day will be ranked as: Low GI $\leq 55$, Medium GI $56-69$ and High GI $\geq 70$. Similarly GL per day will be ranked as: Low GL $<80$, Medium GL 80 120 and a high GL >120 [32]. Dietary gluten evaluation will be performed by using the Osborne formula for all subjects at baseline. Twenty ppm will be applied to the GFD arm as per Health Canada's definition of GF claims.

A typical day intake is incorporated into the dietary interview at visits 2 and 4 (3 and 9 month visit respectively). All records will be assessed for dietary exclusion or inclusion of gluten. At visits 2-5 (3,6,9, and 12 months) inclusive all participants will complete with the dietitian a 'self-report' questionnaire designed to assess their comfort (both groups) and understanding of their assigned diet.

\section{Height, weight, BMI and z-scores}

Regular monitoring of anthropometric parameters will be performed to assess safety. Height and weight will be obtained at every clinic visit and BMI and z-scores will be derived from those measurements. Level of activity will be ranked as per The Canadian Physical activity guidelines (www.csep.ca/) (Fig. 1).

\section{Dietary adherence}

Dietary adherence will be assessed at each visit following the baseline visit. Adherence assessment will be classified as objective and subjective as described earlier.
Subjective and objective assessment of adherence will include food records collected at visits 1,3 and 5 (randomization, 6 and 12 months) and a typical day intake at visits 2 and 4 (3 and 9 months) for both groups. All records will be evaluated for inclusion and exclusion of gluten. In addition, food records will be analyzed via ESHA, dietary food processor. GI/ GL and gluten content will be assessed at baseline, 6 and 12 months in both arms. For the intervention group, the adherence to GFD will be further assessed by the application of the Celiac Dietary Adherence Test (CDAT). Additionally, TTG-IgA titers will be measured at 6 (Visit 3) and 12 months (Visit 5) visits to asses GFD/GCD compliance.

After randomization (visit 1 ) at visits 2 to 5 , both study groups, intervention and control, will be interviewed to assess and document adverse events including severe hypoglycemia requiring assistance, hospitalization, any changes in non-insulin medications, supplements and vitamins from previous visit. HbA1c and TTG will be collected at visits 1,3 and 5 for both arms. GISS and CDAT questionnaires will also be completed only for intervention group, at each visit.

A trained dietitian from the leading site will train identified dietitians from the partner sites using standardized educational material and a detailed manual of operations describing the goals objectives of the dietary curriculum and the steps for the dietary interview at each visit. The standardized tools will be used to maintain quality and consistency of dietary education and collection of information.

\section{Intervention group}

Visit 1: The dietary interview will consist of a comprehensive nutritional assessment followed by the GFD education. "Your Guide to Well-being: Managing Celiac Disease and Diabetes" is a resource manual, divided into modules, that was specifically designed for this population to address the complex dietary nature of living with both CD and T1D. This resource as well as "Pocket Dictionary of Ingredients" and 'Checking Ingredient List' by the Canadian Celiac Association will be provided to the participants along with the GFD education. Participants will be directed to online resources such as Apps that will help and guide them in determining presence of gluten in food, as well as locations of gluten free restaurants and stores in their vicinity. At the end of the education session an assessment will be carried out using food labels for the comprehension of the GFD, carb counting skills and current diabetes management. Three day food records will be collected, clarified and sent to central analysis for ESHA (Elizabeth Stewart Hands and Associates) processing. 


\section{Control group}

Visit 1: The dietary interview will consist of a comprehensive nutritional assessment including on how well the subjects are managing their current diet and ensuring consumption of GC foods. Food labels will be used to assess carbohydrate counting skills. Three day food records will be collected, clarified and sent to central analysis for ESHA processing.

A 4 week follow-up call will be done in both groups to support, address questions and concerns, document any changes in non-insulin medications, supplements and vitamins and document adverse events including severe hypoglycemia requiring assistance.

\section{Follow-up visits 2, 3 and 4 (Months 3, 6 and 9) Intervention group}

The dietary interview will address any gaps in education; provide nutritional support and reinforcement of GFD topics and modules, as required. Subject's food records analysis from visit 1 will be reviewed for imbalances and deficiencies in macronutrients as per Canadian Diabetes Association guidelines and against DRIs. A plan to address any issues or concerns will be implemented and a "Typical Day Intake" will be performed to assess for gluten exclusion and/or inclusion. Subjects with the dietitian will complete a 'self-report' questionnaire designed to assess their comfort with their assigned diet. The dietitian will rank the subjects adherence using specific criteria, see Fig 3. Three day food records will be provided for visit 3 .

\section{Control group}

The dietitian interview will aim to support current dietary management. Subject's food records analysis from visit 1 will be reviewed for imbalances and deficiencies; a plan to address any issues or concerns will be implemented. A "Typical Day Intake" will be performed to assess for gluten exclusion and/or inclusion. Subjects with the dietitian will complete a 'self-report' questionnaire designed to assess their comfort with their assigned diet. The dietitian will rank the subjects adherence using specific criteria, see Fig 3. Three day food records will be provided for visit 3.

At 3 (Visit 2) and 9 months (Visit 4) a "Typical Day Intake" interview will be performed as part of the diet interview and food records will be provided for next visit. At visit 3 and 5 (6 and 12 months) in addition to the dietary interview, food records will be collected and sent to central analysis for ESHA processing. Handouts specific to each group (GFD/GCD) have been designed to address the possible dietary deficiencies or imbalances in macronutrients as well as fiber, iron, calcium and vita$\min \mathrm{D}$.

\section{Conditional telephone contact (Month 7)}

This telephone call will apply to subjects who are found to be non-adherent (as per TTG IgA results from Visit 3 ) to their assigned diet. For both groups: dietitian will address follow-up questions and concerns, barriers to dietary adherence, document any changes in insulin regimen or in non-insulin medications, supplements and vitamins, document adverse events including severe hypoglycemia requiring assistance.

\section{Visit 5 (Month 12)}

For both arms this visit will entail all of the details as per visit 3 (month 6) visit. The dietitian will conduct an exit interview consisting of 7 questions for GFD and 4 questions for the GCD. Subjects assigned to the GCD will be asked if they would like to follow the GFD.

\section{Outcome assessments}

\section{CDAT and TTG and Dietitian's ranking}

We anticipate that the subjective ranking by the dietitian will correlate with the subject's TTG, CDAT and selfreport in both arms. We also anticipate that the average GI and the GL content of the GFD will be higher than the GCD due to the nature of some of the GF foods which tend to be more refined higher in carbohydrate and contain less fiber than the GC equivalents. The difference in GI and GL between the groups may also explain the possible BG variability in both groups as will be documented with (continuous glucose monitoring (CGM)).

\section{Changes in dietary intervention assignment (cross-overs)}

In cases of symptom presentation as defined by Mahmud et al. [28] subjects will be crossed over into the GFD.

Power calculation and statistics:

The outcome measure used to calculate sample size will be the change in HbA1c level from baseline to month 12 . Based on previous work, in a non-randomized comparison of $\mathrm{HbA1c}$ levels over time in type 1 diabetes patients with asymptomatic CD who received a GFD versus patients with type 1 diabetes alone, an increase in HbAlc of $8.3 \pm 1.1 \%$ to $8.9 \pm 1.5 \%$ was observed.10 As such, 91 evaluable individuals per group are required to provide $80 \%$ power to detect a difference of at least $0.5 \%$ at the 0.05 level of significance. The enrolment of 200 participants will allow for a non-evaluable rate of about $10 \%$. To reach the recruitment target of 200 individuals, approximately 5000 patients with an established diagnosis of type 1 diabetes will be screened for asymptomatic $\mathrm{CD}$. Efficacy analyses will be performed on the intent-to-treat population consisting of all randomized participants.

The primary efficacy outcome, the change in HbA1c at 12 months, will be analyzed using an analysis of covariance (ANCOVA) model that includes adjustment for 
baseline HbA1c, age groups and investigative sites. Treatment effect will be quantified using the point estimate, the two-sided $95 \% \mathrm{CI}$, and associated p value. Significant effect will be declared at the $5 \%$ significance level.

Severe hypoglycemic episodes over time will be analyzed as Poisson counts using the generalized estimating equations approach to account for correlation among repeated measures within an individual. The QOL data over time will be analyzed by means of a linear mixed effects model including factors for treatment, investigative sites, age groups, and baseline QOL scores. BG variability and change in Z-score at the lumbar spine will be analyzed using the ANCOVA approach. The Statistical Analysis System (SAS) procedures GENMOD, MIXED and GLM in SAS V.9.2, will be used to perform the respective analyses.

Patient consent: Obtained.

Ethics Approval: Research Ethics Board Hospital for Sick Children, University of Toronto and at all recruitment sites.

The CD-DIET study data safety and monitoring board will perform regular reviews of collected safety and efficacy data. The protocol was peer reviewed by the JDRF.

\section{Trial organization}

- JDRF-CCTN (Juvenile Diabetes Research Foundation - Canadian Clinical Trials Network) financial and organizational support

- Robarts Clinical Trials and Sickkids - Monitoring, safety and adverse events coordination

- Group Organization - Paediatric and adult endocrinologists, gastroenterologists, diabetes dietitians, and research coordinators in each of different sites.

\section{Discussion and dissemination}

The CD-DIET study is a large scale clinical trial that will further contribute to the pool of knowledge and novel implications to the treatment of asymptomatic celiac disease and TID. The dietary curriculum will be coordinated across all centers by the main site dietitian, using standardized educational materials and a manual of operations. Both pediatric and adult patients will be randomized to a GFD or GCD. Adherence to the diets will be determined from the dietary interview, TTG-IgA blood tests and CDAT (GFD) and subjects' self-report. CDAT is highly correlated with Standardized Dietician Evaluation (SDE) and even superior to TTG IgA serology with regards to GFD adherence [31]. The Canadian context to the study is important, as the 2013 Canadian Diabetes Association's clinical practice guidelines suggests a targeted symptom based screening approach [33]. While the literature supports the implementation of a GFD in symptomatic CD and T1D patients, it is less clear as to the benefits for asymptomatic patients. Our curriculum was established in concurrence with the current literature and is designed to address a number of issues: the limited educational literature the challenges associated with dietary quality and adherence and the implicated social challenges of a GF lifestyle in addition to T1D and. Participants will be supported by dietitians throughout the study and subjects on the intervention group and later the control group will have to have access to 'Your Guide to Well-being: Managing Celiac disease and Diabetes' a resource specifically designed to address the nutritional intricacies and related challenges of both conditions: $\mathrm{CD}$ and T1D.

The dietary quality of the GFD will be assessed through food records and interviews. The literature reports that GF equivalents tend to be lower in micronutrients and fibre, but higher in calories, fat and sodium $[5,13]$. The participant's diets will be evaluated for deficiencies and imbalances of macronutrients and micronutrients. We will assess the quantity and quality of carbohydrate intake using the glycemic index as well as glycemic load and calculate dietary gluten in our subject population. Dietary gluten quantification was previously demonstrated in Europe and applied to one of our smaller pilot studies with some limitations inherent to methodology and with the Canadian and American nutrient databases. We have described a method to help apply the Osborne calculation in North America [11] and plan to correlate adherence to gluten consumption and describe our population's consumption patterns as it relates to $\mathrm{CD}$ and T1D.

Participants will also have the opportunity to report their adherence to the diet that they are randomized to through a 'self-report' questionnaire from 'strict' to 'partial' to 'non' adherent [34]. Although some studies show that patients overestimate adherence to GFD and that reliability of self-reports decreases with time on a GFD $[16,35]$, our previous findings show a close correlation between self-reports and dietitians' assessment [36].

One of the greater challenges with asymptomatic patients if that they report better QOL and health prior to diagnosis [25]. This is likely due to the perceived burden of novel dietary restriction.

This study will answer some of the questions with regards to perceived benefits of GFD, especially when almost a third [37] of health practitioners are unsure whether they should be recommending GFD to asymptomatic patients [38]. The study was initiated in 2012, now active at 16 centers, and it is anticipated that results will be available by 2016 .

\section{Abbreviations}

CCA: Canadian Celiac Association; CD: Celiac Disease; CDA: Canadian Diabetes Association; CDAT: Celiac Dietary Adherence Test; CD-DIET Study: Celiac disease and Diabetes- Dietary Intervention and Evaluation Trial; 
CPG: Clinical Practice Guidelines; GCD: Gluten containing diet; GFD: Gluten free diet; GISS: Gastrointestinal symptom scale; HbA1c: Glycosylated hemoglobin; PedsQL: Pediatric Quality of Life; QOL: Quality of life; SDE: Standardized Dietitian Evaluation; T1D: Type 1 Diabetes; TTG: Tissue Transglutaminase antibodies; TTG - IgA: Tissue Transglutaminase IgA Antibody Assay.

\section{Competing interests}

The authors declare that they have no competing interests.

\section{Author's contribution}

EA conceived the dietary design of this study, led and trained its dietitians, participated in data acquisition and drafted the original dietary manuscript. MAM, NH, MF, and TC participated in manuscript review. FHM as the lead principle investigator participated in the conception of the dietary design data acquisition and drafted the initial dietary manuscript. All authors read and approved the final manuscript.

\section{Acknowledgements}

Karima Noordin, Kamaljeet Sahota, Jolie Davies-Shaw, Alessia Kazakova \& Antoine Clarke participated in study co-ordination data acquisition. All of the CD-DIET investigators, collaborators and research staff engaed in the trial.

\section{Funding}

This work is supported by Juvenile Diabetes Research FoundationCanadian Clinical Trials Network (JDRF-CCTN) grant number (CCTN 1103) and receives operating support from Robarts Clinical Trials Inc. for data monitoring, quality assurance and adverse events coordination. Trial Registration Number: NCT01566110. Date of Registration: March, 2012.

\section{Author details}

'Department of Pediatrics, Division of Endocrinology, Hospital for Sick Children, University of Toronto, Toronto, ON, Canada. ${ }^{2}$ Division of Gastroenterology, Hepatology and Nutrition, Hospital for Sick Children, University of Toronto, Toronto, ON, Canada. ${ }^{3}$ Division of Endocrinology Paediatrics, London Health Sciences Centre, London, ON, Canada. ${ }^{4}$ Division of Endocrinology, Markham Stouffville Hospital, Toronto, ON, Canada. ${ }^{5}$ Division of Endocrinology and Metabolism, Children's Hospital of Eastern Ontario, Ottawa, ON, Canada.

\section{Received: 6 November 2015 Accepted: 14 December 2015}

\section{Published online: 21 December 2015}

\section{References}

1. Ferretti G, Bacchetti T, Masciangelo S, Saturni L. Celiac disease, inflammation and oxidative damage: a nutrigenetic approach. Nutrients. 2012;4(4):243-57. PubMed Pubmed Central PMCID: Pmc3347005, Epub 2012/05/19. eng.

2. Farrell RJ, Kelly CP. Celiac sprue. N Engl J Med. 2002;346(3):180-8. PubMed Epub 2002/01/18. eng.

3. Sud S, Marcon M, Assor E, Palmert MR, Daneman D, Mahmud FH. Celiac disease and pediatric type 1 diabetes: diagnostic and treatment dilemmas. Int J Pediatr Endocrinol. 2010;2010:161285. PubMed Pubmed Central PMCID: 2905696, Epub 2010/07/24. eng

4. Mahmud FH, Murray JA, Kudva YC, Zinsmeister AR, Dierkhising RA, Lahr BD, et al. Celiac disease in type 1 diabetes mellitus in a North American community: prevalence, serologic screening, and clinical features. Mayo Clin Proc. 2005:80(11):1429-34. PubMed Pubmed Central PMCID: 2128722, Epub 2005/11/22. eng.

5. Wild D, Robins GG, Burley VJ, Howdle PD. Evidence of high sugar intake, and low fibre and mineral intake, in the gluten-free diet. Aliment Pharmacol Ther. 2010;32(4):573-81. PubMed Epub 2010/06/10. eng.

6. Saturni L, Ferretti G, Bacchetti T. The gluten-free diet: safety and nutritional quality. Nutrients. 2010;2(1):16-34. PubMed Pubmed Central PMCID: 3257612, Epub 2010/01/01. eng.

7. Shepherd SJ, Gibson PR. Nutritional inadequacies of the gluten-free diet in both recently-diagnosed and long-term patients with coeliac disease. J Hum Nutr Diet. 2013:26(4):349-58. PubMed Epub 2012/12/04. eng.

8. Ohlund K, Olsson C, Hernell O, Ohlund I. Dietary shortcomings in children on a gluten-free diet. J Hum Nutr Diet. 2010;23(3):294-300. PubMed Epub 2010/03/27. eng.
9. Kulai TM, Rashid MMMFC. Assessment of Nutritional Adequacy of Packaged Gluten-free Food Products. Can J Diet Pract Res. 2014;75(4):186-90. PubMed Epub 2015/06/13. Eng.

10. Thompson T, Dennis M, Higgins LA, Lee AR, Sharrett MK. Gluten-free diet survey: are Americans with coeliac disease consuming recommended amounts of fibre, iron, calcium and grain foods? J Hum Nutr Diet. 2005; 18(3):163-9. PubMed Epub 2005/05/11. eng

11. Ahola AJ, Mikkila V, Makimattila S, Forsblom C, Freese R, Groop PH. Energy and nutrient intakes and adherence to dietary guidelines among Finnish adults with type 1 diabetes. Ann Med. 2012;44(1):73-81. PubMed Epub 2010/11/05. eng.

12. Mehta SN, Volkening LK, Quinn N, Laffel LM. Intensively managed young children with type 1 diabetes consume high-fat, low-fiber diets similar to age-matched controls. Nutr Res. 2014;34(5):428-35. PubMed Central PMCID: 4096954, Epub 2014/06/12. eng.

13. Miranda J, Lasa A, Bustamante MA, Churruca I, Simon E. Nutritional differences between a gluten-free diet and a diet containing equivalent products with gluten. Plant Foods Hum Nutr. 2014;69(2):182-7. PubMed Epub 2014/03/01. eng.

14. Gambus H, Gambus F, Pastuszka D, Wrona P, Ziobro R, Sabat R, et al. Quality of gluten-free supplemented cakes and biscuits. Int J Food Sci Nutr. 2009;60 Suppl 4:31-50. PubMed Epub 2009/03/31. eng.

15. Lee A, Newman JM. Celiac diet: its impact on quality of life. J Am Diet Assoc. 2003;103(11):1533-5. PubMed Epub 2003/10/25. eng.

16. Leffler DA, Edwards-George J, Dennis M, Schuppan D, Cook F, Franko DL, et al. Factors that influence adherence to a gluten-free diet in adults with celiac disease. Dig Dis Sci. 2008;53(6):1573-81. PubMed Pubmed Central PMCID: 3756800, Epub 2007/11/09. eng.

17. Barratt SM, Leeds JS, Sanders DS. Quality of life in Coeliac Disease is determined by perceived degree of difficulty adhering to a gluten-free diet, not the level of dietary adherence ultimately achieved. J Gastrointestin Liver Dis. 2011:20(3):241-5. PubMed Epub 2011/10/01. eng.

18. Rashid M, Cranney A, Zarkadas M, Graham ID, Switzer C, Case S, et al. Celiac disease: evaluation of the diagnosis and dietary compliance in Canadian children. Pediatrics. 2005;116(6):e754-9. PubMed Epub 2005/12/03. eng.

19. Biagetti C, Naspi G, Catassi C. Health-related quality of life in children with celiac disease: a study based on the Critical Incident Technique. Nutrients. 2013:5(11):4476-85. PubMed Pubmed Central PMCID: 3847743, Epub 2013/ 11/15. eng.

20. Whitaker JKH, West J, Holmes GKT, Logan RFA. Patient perceptions of the burden of coeliac disease and its treatment in the UK. Aliment Pharmacol Ther. 2009;29(10):1131-6.

21. Altobelli E, Paduano R, Gentile T, Caloisi C, Marziliano C, Necozione S, et al. Health-related quality of life in children and adolescents with celiac disease: survey of a population from central Italy. Health Qual Life Outcomes. 2013;11:204. PubMed Pubmed Central PMCID: 3878970, Epub 2013/12/07. eng.

22. Nordyke K, Norstrom F, Lindholm L, Stenlund H, Rosen A, Ivarsson A. Health-related quality of life in adolescents with screening-detected celiac disease, before and one year after diagnosis and initiation of gluten-free diet, a prospective nested case-referent study. BMC Public Health. 2013:13: 142. PubMed Pubmed Central PMCID: 3585471, Epub 2013/02/19. eng.

23. van Koppen EJ, Schweizer JJ, Csizmadia CG, Krom Y, Hylkema HB, van Geel $\mathrm{AM}$, et al. Long-term health and quality-of-life consequences of mass screening for childhood celiac disease: a 10-year follow-up study. Pediatrics. 2009;123(4):e582-8. PubMed Epub 2009/04/02. eng.

24. Bystrom IM, Hollen E, Falth-Magnusson K, Johansson A. Health-related quality of life in children and adolescents with celiac disease: from the perspectives of children and parents. Gastroenterol Res Pract. 2012;2012. 986475. PubMed Pubmed Central PMCID: 3324145, Epub 2012/05/02. eng

25. Ukkola A, Maki M, Kurppa K, Collin P, Huhtala H, Kekkonen L, et al. Diet improves perception of health and well-being in symptomatic, but not asymptomatic, patients with celiac disease. Clin Gastroenterol Hepatol. 2011 9(2):118-23. PubMed Epub 2010/10/30. eng.

26. Pulido O, Zarkadas M, Dubois S, Macisaac K, Cantin I, La Vieille S, et al. Clinical features and symptom recovery on a gluten-free diet in Canadian adults with celiac disease. Can J Gastroenterol. 2013;27(8):449-53. PubMed Pubmed Central PMCID: 3956033, Epub 2013/08/13. eng.

27. Mahmud FH, De Melo EN, Noordin K, Assor E, Sahota K, Davies-Shaw J, et al. The Celiac Disease and Diabetes-Dietary Intervention and Evaluation Trial (CD-DIET) protocol: a randomised controlled study to evaluate treatment of 
asymptomatic coeliac disease in type 1 diabetes. BMJ open. 2015;5(5), e008097. PubMed Pubmed Central PMCID: PMC4431067, Epub 2015/05/15. eng.

28. Hall NJ, Rubin G, Charnock A. Systematic review: adherence to a gluten-free diet in adult patients with coeliac disease. Aliment Pharmacol Ther. 2009; 30(4):315-30. PubMed Epub 2009/06/03. eng.

29. Assor E, Davies-Shaw J, Marcon MA, Mahmud FH. Estimation of Dietary Gluten Content using Total Protein in Relation to Gold Standard Testing in a Variety of Foods. J Nutr Food Sci. 2014;4(296). doi:10.4172/2155-9600. 1000296.

30. Canada H. Health Canada's Position on Gluten Free Claims. 2012.

31. Leffler DA, Dennis M, Edwards George JB, Jamma S, Magge S, Cook EF, et al. A simple validated gluten-free diet adherence survey for adults with celiac disease. Clin Gastroenterol Hepatol. 2009;7(5):530-6. 6 e1-2. PubMed Epub 2009/03/10. eng.

32. Dietary Carbohydrates [Internet]. 2015. Available from: http://www.uptodate com/contents/dietary-carbohydrates.. Accessed 21 Oct 2015.

33. Wherrett D, Huot C, Mitchell B, Pacaud D. Type 1 diabetes in children and adolescents. Can J Diabetes. 2013;37 Suppl 1:S153-62. PubMed Epub 2014/ 05/16. eng.

34. Fera T, Cascio B, Angelini G, Martini S, Guidetti CS. Affective disorders and quality of life in adult coeliac disease patients on a gluten-free diet. Eur J Gastroenterol Hepatol. 2003;15(12):1287-92. PubMed Epub 2003/11/19. eng.

35. Leffler DA, Edwards George JB, Dennis M, Cook EF, Schuppan D, Kelly CP. A prospective comparative study of five measures of gluten-free diet adherence in adults with coeliac disease. Aliment Pharmacol Ther. 2007; 26(9):1227-35. PubMed Epub 2007/10/20. eng.

36. Sud S, Marcon M, Assor E, Daneman D, Mahmud FH. Quality of life in children with diabetes and celiac disease: minimal impact of the 'double diagnosis'. Pediatr Diabetes. 2012;13(2):163-9. PubMed Epub 2011/06/16. eng

37. Sud S, Marcon M, Assor E, Daneman D, Mahmud FH. Health Professional Attitudes Regarding Celiac Disease Screening and Treatment in Pediatric Type 1 Diabetes. Canadian J Diabetes. 2011;35(4):334-9.

38. Taler I, Phillip M, Lebenthal Y, de Vries L, Shamir R, Shalitin S. Growth and metabolic control in patients with type 1 diabetes and celiac disease: a longitudinal observational case-control study. Pediatr Diabetes. 2012;13(8): 597-606. PubMed Epub 2012/05/09. eng.

\section{Submit your next manuscript to BioMed Central and we will help you at every step:}

- We accept pre-submission inquiries

- Our selector tool helps you to find the most relevant journal

- We provide round the clock customer support

- Convenient online submission

- Thorough peer review

- Inclusion in PubMed and all major indexing services

- Maximum visibility for your research

Submit your manuscript at www.biomedcentral.com/submit

C) Biomed Central 